\title{
Comparison Between the Jugular and Subclavian Vein as Insertion Site for Central Venous Catheters: Microbiological Aspects and Risk Factors for Colonization and Infection
}

\author{
Geraldo Sadoyama and Paulo Pinto Gontijo Filho
}

\author{
Laboratory of Microbiology - ARIMP - Federal \\ University of Uberlândia, Uberlândia/MG, Brazil
}

\begin{abstract}
Bacterial counts were made of catheter insertion site and of catheter tips to help determine risk factors associated with catheterization of the jugular and subclavian veins. Among the 116 patients included in this study, $69 \%$ had central venous catheters (CVC) in the subclavian vein. Seven or more days catheterization $(p=0.001)$ and $\geq 3$ invasive devices $(p=0.01)$ were infection risk factors associated with catheterization of the jugular vein. More than half of the patients presented high colony counts at the insertion site $\left(\geq 200 \mathrm{CFU} / 20 \mathrm{~cm}^{2}\right)$ and $27 \%$ of the catheter tips were contaminated. The risk factors associated with contaminated catheter tips were $\geq 14$ days hospital stay $(p=0.02), \geq 7$ days catheterization $(p=0.01)$ and antibiotic therapy $(p=0.04)$. Coagulase-negative staphylococci (CoNS) and Staphylococcus aureus were the most common microorganisms at the insertion site $(\mathbf{7 8 \%})$ and in the catheter tip $(94 \%)$. Five patients presented sepsis $(4.1 \%)$, four caused by Staphylococci and one by GNB. Twelve patients had the same microorganisms at the insertion site and catheter tip. We found a high prevalence of ORSA $(62.5 \%)$ and ORCoNS $(57.1 \%)$ in catheter tips. The high counts of staphylococci, including ORSA and ORCoNS, at the insertion site, and the significant association of this colonization with catheter tip contamination, indicate that the skin is an important reservoir of microorganisms associated with catheter-related bloodstream infection (CR-BSI). Health professionals should be aware of this potential source of infection at the CVC insertion site.

Key Words: CVC, risk factor, CR-BSI, ORSA, ORCoNS.
\end{abstract}

Infection is a potentially life-threatening complication of central venous catheter (CVC). Many factors may contribute to the contamination of CVC [01]. Multivariate analyses of prospective studies on risk factors for central venous related infection rank heavy insertion-site colonization as one of the most important factors [02].

Contamination of CVC is a common problem in intensive care units. Several studies have reported Received on 07 August 2002; revised 11 September 2002. Address for correspondence: Dr. Geraldo Sadoyama, MSc. Area of Immunology, Microbiology and Parasitology, Laboratory of Microbiology, Block 4C, Campus Umuarama, Federal University of Uberlândia, Uberlândia, MG, Brazil. 38400-902. Phone : (34)3218-2236; Fax number: (34)3218-2332.

E-mail: sadoyama@umuarama.ufu.br

The Brazilian Journal of Infectious Diseases 2003;7(2):142-148 (C) 2003 by The Brazilian Journal of Infectious Diseases and Contexto Publishing. All rights reserved. contamination or colonization rates of catheters to range between $3.8 \%$ and $4.7 \%$ [01] and incidences of catheter-related bloodstream infections (CR-BSI) for short-term, noncuffed, central venous catheters range from $3 \%$ to $5 \%$, which is much higher than that reported for peripheral intravenous catheters [03].

We qualitatively and quantitatively evaluated contamination with microorganisms at the skin insertion site of CVC (subclavian or jugular vein), of catheter tips, as well as sepsis and other risk factors associated with these procedures.

\section{Materials and Methods}

A prospective observational study of nontunneled CVC (116 patients) over a 11-month period was conducted from September 2000 to June 2001 at the Clinical Surgical II Ward (33 beds) and the Intensive 
Care Unit ( 9 beds) of the Uberlândia Federal University Hospital (HC-UFU).

All the CVCs (vialon biomaterial - Becton Dickinson $\left({ }^{\circledR}\right)$ followed in this trial were placed by cannulation, without using the Seldinger technique, in the jugular or subclavian vein by attending physicians or supervised medical students; they used aseptic technique for insertion, with sterile gloves, drapes, gowns, face masks and surgical scrubbing with povidine-iodine. The option for vascular access (jugular $\mathrm{x}$ subclavian) was decided by the surgeons/physicians/ supervised medical students based on experience, without considering the severity of the case. After the introduction of the CVC, the skin insertion site was cleaned with alcoholic povidine iodine solution daily. The insertion site was inspected every 24 hours for evidence of infection, such as erythema, purulence, swelling, or tenderness near the catheter. The catheter remained in place either until it was no longer needed, or when a specific event, such as a possible catheterrelated infection, made removal necessary.

The skin at the insert site of CVC was initially swabbed. Two samples were taken: the first, when the catheter was inserted, and the second 5-7 days after insertion. Approximately $20 \mathrm{~cm}^{2}$ of skin at the catheter insertion site was cleaned with sterile pre-moistened swabs. Each swab was then inoculated on both blood agar and salted mannitol agar. A skin culture was considered positive if $\geq 200$ colony forming units (CFU) were isolated [04]. Catheters were removed under sterile conditions. The tips of the catheters were cut with sterile scissors and transported to the laboratory in tubes containing $10 \mathrm{~mL}$ of phosphate-buffered saline (PBS) $+0.1 \%$ Tween 80 . The cultures of the catheter tips were conducted quantitatively, using a modified Brun-Buisson technique: a segment of approximately $5 \mathrm{~cm}$ of the catheter tip was placed in a tube containing $10 \mathrm{~mL}$ of PBS $+0.1 \%$ Tween 80 and agitated with a vortex for 1 minute; $0.1 \mathrm{~mL}$ of the liquid was inoculated on blood agar, McConkey agar, and mannitol salt agar [05]. The cultures were considered positive if $\geq 10^{2}$ $\mathrm{CFU} / \mathrm{mL}$ were isolated. The clinical specimens obtained from the skin and from the tip of the CVC were identified by traditional techniques. They were initially classified as Gram-negative bacilli (GNB), Grampositive bacilli (GPB), Gram-positive cocci or yeast fungi by Gram stain. The Gram-positive cocci were submitted to supplemental tests, such as: catalase, coagulase, fermentation of the mannitol and DNAse, and were separated into $S$. aureus, coagulase-negative staphylococci (CoNS) and other Gram-positive cocci. The blood specimens were obtained by peripheral venous puncture. The blood cultures were made by inoculating 5-10 mL of blood in a Bactec/Alert ${ }^{\circledR}$ (Organon Teknika Corp.). bottle, an automated commercial system Samples were identified by traditional phenotypic tests.

A catheter-related bloodstream infection (CR-BSI) was defined as: isolation of the same microorganism (i.e., identical species and type of resistance) from a semiquantitative or quantitative culture of a catheter segment and from the blood (preferably drawn from a peripheral vein) of a patient with accompanying clinical symptoms and no other source of infection.

All Staphylococcus isolates were plated on MuellerHinton agar, supplemented with $4 \% \mathrm{NaCl}$ and oxacillin $(6 \mu \mathrm{g} / \mathrm{mL})$. The inoculum size was adjusted to yield a final inoculum of approximately $10^{5} \mathrm{CFU} / \mathrm{mL}$. Oxacillin resistance was confirmed by surface growth, after incubation for $24 \mathrm{~h}$ at $30^{\circ}-35^{\circ} \mathrm{C}$ [06]. Statistical significance was defined by a $p$ value less than 0.05 . The frequencies of qualitative variables were compared using a chi-squared test with Yates' correction or Fisher's Exact Test (two-tailed). Epi Info 2000 Software (Centers for Disease Control and Prevention, Atlanta, GA) was used to perform the statistical analyses.

\section{Results}

Of the 116 patients included in this study, 43 were hospitalized in the Intensive Care Unit and 73 in the Clinical Surgical II Ward. We were able to recuperate just half (50.9\%) of the catheter tips. High rates of contamination at the catheter insertion site were found in about half of the patients. Most patients were catheterized in the subclavian vein (69\%). The risk factors $\geq 7$ days catheterization $(\mathrm{p}=0.001 ; \mathrm{RR}=2.92)$ 
Table 1. Risk factors associated with central venous insertion of catheters in the jugular and subclavian vein

\begin{tabular}{|c|c|c|c|}
\hline \multirow[t]{2}{*}{ Risk factors } & \multicolumn{3}{|c|}{ Insertion site } \\
\hline & $\begin{array}{c}\text { Jugular } \\
\mathbf{N}=\mathbf{3 6}(\%)\end{array}$ & $\begin{array}{c}\text { Subclavian } \\
\mathrm{N}=\mathbf{8 0}(\%)\end{array}$ & $\mathbf{p}(\mathbf{R R})$ \\
\hline Insertion site $\geq 200 \mathrm{CFU} / 20 \mathrm{~cm}^{2}$ & $21(58.3)$ & $38(47.5)$ & $0.49(1.28)$ \\
\hline Age $\geq 60$ years & $9(25.0)$ & $26(32.5)$ & $0.59(0.78)$ \\
\hline$\geq 14$ days hospital stay & $25(69.4)$ & $50(62.5)$ & $0.60(1.24)$ \\
\hline$\geq 7$ days catheterization & $26(72.2)$ & $35(43.7)$ & $0.01(2.22)$ \\
\hline ICU- Care & $17(47.2)$ & $26(32.5)$ & $0.19(1.52)$ \\
\hline Surgical Ward & $19(52.8)$ & $54(67.5)$ & $0.19(0.66)$ \\
\hline $\mathrm{TPN}^{2}$ & $11(30.5)$ & $21(26.2)$ & $0.79(1.16)$ \\
\hline Antibiotic therapy & $21(58.3)$ & $32(40.0)$ & $0.10(1.66)$ \\
\hline$\geq 3$ antibiotics & $10(27.8)$ & $15(18.7)$ & $0.39(1.40)$ \\
\hline Other invasive devices & $33(91.7)$ & $68(85.0)$ & $0.38(1.63)$ \\
\hline$\geq 3$ devices & $22(61.1)$ & $31(38.7)$ & $0.04(1.87)$ \\
\hline Mortality & $2 \quad(5.5)$ & $1 \quad(1,2)$ & $0.22 \quad(2.2)$ \\
\hline
\end{tabular}

ICU: intensive care unit; TPN: total parenteral nutrition.

Table 2. Relationship of bacterial contamination between insertion site and catheter tip

\begin{tabular}{lcc}
\hline & \multicolumn{2}{c}{ Catheter tip } \\
\cline { 2 - 3 } $\begin{array}{l}\text { Insertion Site } \\
\left(\text { CFU/20 } \mathbf{c m}^{\mathbf{2}}\right)\end{array}$ & $\begin{array}{c}\text { Negative } \\
\mathbf{N}=\mathbf{4 3}(\boldsymbol{\%})\end{array}$ & $\begin{array}{c}\text { Positive } \\
\mathbf{N}=\mathbf{1 6}(\boldsymbol{\%})\end{array}$ \\
\hline$<200 \mathrm{CFU}$ & $26(60.5)$ & $04(25.0)$ \\
$\geq 200 \mathrm{CFU}$ & $17(39.5)$ & $12(75.0)$ \\
\hline $\mathrm{p}=0.03(\mathrm{RR}=1.48)$. & &
\end{tabular}

Table 3. Risk factors associated with colonization in the catheter tip

\begin{tabular}{lrrr}
\hline Risk Factors & \multicolumn{3}{c}{ Catheter tip } \\
\cline { 2 - 4 } & $\begin{array}{c}\text { Positive } \\
\mathbf{N = 1 6}(\%)\end{array}$ & $\begin{array}{c}\text { Negative } \\
\mathbf{N = 4 3}(\%)\end{array}$ & p (RR) \\
\hline Dressing $^{\text {Age }}{ }^{3}$ 60 years & $2(12.5)$ & $6(13.9)$ & $1.0(0.91)$ \\
Hospital stay $^{3}$ 14 days & $8(50.0)$ & $18(41.9)$ & $0.79(1.27)$ \\
Catheterization $^{3}$ 7 days & $15(93.7)$ & $26(60.5)$ & $0.02(6.59)$ \\
Antibiotic therapy $^{\geq 3 \text { antibiotics }}$ & $15(93.7)$ & $23(53.5)$ & $0.01(8.29)$ \\
Other invasive devices & $15(93.7)$ & $28(65.1)$ & $0.04(5.58)$ \\
$\geq 3$ devices & $06(37.5)$ & $05(11.6)$ & $0.05(2.62)$ \\
\hline
\end{tabular}

* not calculated 
Table 4. Microorganisms colonizing the insertion site of the CVCs in the jugular and subclavian vein

\begin{tabular}{lccc}
\hline Microorganisms & \multicolumn{3}{c}{ Insertion site } \\
\cline { 2 - 5 } & $\begin{array}{c}\text { Jugular } \\
\mathbf{N = 3 6}(\%)\end{array}$ & $\begin{array}{c}\text { Subclavian } \\
\mathbf{N = 8 0}(\%)\end{array}$ & p (RR) \\
\hline Gram-positive cocci & $30(83.3)$ & $74(92.5)$ & $0.24(0.58)$ \\
Staphylococci & $27(75.0)$ & $69(86.3)$ & $0.22(0.63)$ \\
Other cocci & $03(8.3)$ & $05(6.2)$ & $0.70(1.23)$ \\
Gram-negative bacilli & $06(16.7)$ & $03(3.8)$ & $0.04(2.34)$ \\
Yeasts & $08(22.2)$ & $04(5.0)$ & $0.007(2.69)$ \\
Others & $01(2.8)$ & $02(2.5)$ & $1.0 \quad(1.01)$ \\
\hline
\end{tabular}

and the use of $\geq 3$ invasive devices $(\mathrm{p}=0.01 ; \mathrm{RR}=2.09$ ) were gave significantly more contamination in the jugular vein, than in the subclavian vein. No significant differences were found between these two insertion sites in the frequency of microorganisms $(\mathrm{p}=0.49)$, age $(\mathrm{p}=0.59)$, hospital stay $(\mathrm{p}=0.60)$, ICU-care $(0.19)$, surgical ward (0.19), TPN (0.79), antibiotic therapy $(0.10)$ or mortality $(\mathrm{p}=0.22)$ (Table 1$)$. Among the 59 catheter tips that were obtained and processed, about $27 \%$ were contaminated. There was a significant correlation between contamination rates at the insertion site and in catheter tips when there were $\geq 200 \mathrm{CFU} /$ $20 \mathrm{~cm}^{2}$ skin surface at the catheter site $(\mathrm{p}=0.03 ; \mathrm{RR}=1.48)($ Table 2$)$. Bivariate analysis of the risk factors associated with bacterial contamination of the catheter tip demonstrated significant differences in relation to hospital stay $\geq 14$ days ( $\mathrm{p}=0.02 ; \mathrm{RR}=6.59$ ), $\geq 7$ days catheterization $(\mathrm{p}=0.01 ; \mathrm{RR}=8.29)$ and antibiotic therapy $(\mathrm{p}=0.04 ; \mathrm{RR}=5.58)$ (Table 3). The number of microorganisms at the $\mathrm{CVC}$ insertion site in the jugular vein was not significantly different from that of the subclavian vein $(\mathrm{p}=0.49 ; \mathrm{R}=1.28)$. Staphylococci were the most frequent microorganisms in the qualitative analysis of the bacterial contaminations at these sites (jugular $75 \%$ vs. subclavian $86 \%$ ); these differences were not significant $(\mathrm{p}=0.22 ; \mathrm{RR}=0.63$ ). The GNB $(\mathrm{p}=0.03 ; \mathrm{RR}=2.31)$ and yeasts $(\mathrm{p}=0.008 ; \mathrm{RR}=2.48)$ were more frequent at the jugular vein (Table 4), however, there were no differences in the numbers of the most frequently isolated microorganisms between those found on the day of catheter insertion and 5-7 days after insertion. Among the 116 patients, five $(4.3 \%)$ presented sepsis and three of them (2.6\%) had CR-BSI. Staphylococcus aureus was isolated in eight (50\%), five of these samples were ORSA (62.5\%), and seven CoNS (43.7\%); $571 \%$ of the CoNS were resistant to oxacillin. Among the 16 contaminated catheter tips, just one (6.3\%) was GNB. Microorganisms detected at the insertion site and within the catheter tip were the same in 12 of the 16 samples, and in three of these 12 patients, the same microorganism was detected in the blood. There were no differences in the frequency of isolates resistant to oxacillin or to antimicrobial use $(\mathrm{p}=0.52)$ in the patients who had staphylococci at the insert site and in the catheter tip (Table 5).

\section{Discussion}

The risk factors for CVC infections include: the use of a multilumen catheter, hemodialysis, catheterization for $\geq 4$ days, difficulty in catheter insertion, prolonged stay in the ICU before catheter insertion and insertion site contamination [07]. Another study associated CVC infections with: insertion sites contaminated with $>50 \mathrm{CFU}$ of CoNS or contaminated with any other microorganisms or cutaneous contamination of the site with $>10^{2}$ CFU [08].

Half of the patients in our study were contaminated at the insertion site with $\geq 10^{2} \mathrm{CFU}$. This proportion is high compared to the less than $17 \%$ found in other studies [4,9]. The HC-UFU routine does not include 
Table 5. Qualitative and quantitative analysis of Staphylococcus aureus and Staphylococcus spp. at the insertion site, inside the catheter tip and in the blood

\begin{tabular}{|c|c|c|c|c|c|c|c|}
\hline Patient & $\begin{array}{l}\text { Insert } \\
\text { vein }\end{array}$ & $\begin{array}{l}\text { Therapeutic } \\
\text { use of anti- } \\
\text { microbials }\end{array}$ & $\begin{array}{c}\text { Insertion } \\
\text { site }(\geq 200 \\
\text { CFU/20 cm })\end{array}$ & $\begin{array}{l}\underset{\text { Catheter }}{\text { tip }} \\
\geq \mathbf{1 0}^{2} \mathrm{CFU}\end{array}$ & $\begin{array}{c}\text { Microorganism } \\
\text { in the insertion } \\
\text { site }\end{array}$ & $\begin{array}{l}\text { Microorganism } \\
\text { in the catheter } \\
\text { tip }\end{array}$ & $\begin{array}{l}\text { Microorganism } \\
\text { in blood }\end{array}$ \\
\hline 1 & Sub & + & + & + & ORSA $^{1}$ & ORSA & ORSA \\
\hline 2 & Sub & - & + & + & ORCoNS $^{2}$ & ORCoNS & _ \\
\hline 3 & Sub & + & + & + & ORSA/ORCoNS & ORSA & _ \\
\hline 4 & Sub & + & + & + & ORSA/OSSA ${ }^{3}$ & ORSA & - \\
\hline 5 & Jug & + & + & + & OSSA & OSSA & OSSA \\
\hline 6 & Sub & + & + & + & ORCoNS & ORSA & _ \\
\hline 7 & Sub & + & - & + & $\mathrm{ORCoNS}^{4}$ & $\mathrm{GNB}^{5}$ & - \\
\hline 8 & Sub & + & - & + & ORCONS & ORCoNS & - \\
\hline 9 & Jug & + & + & + & OSCoNS & OSCoNS & - \\
\hline 10 & Sub & + & + & + & OSCoNS & ORCoNS & - \\
\hline 11 & Sub & + & + & + & OSSA & OSSA & OSSA \\
\hline 12 & Sub & + & + & + & ORSA/OSCoNS & OSCoNS & _ \\
\hline 13 & Sub & + & + & + & ORCONS/ORSA & ORSA & - \\
\hline 14 & Sub & - & + & + & OSSA & OSSA & - \\
\hline 15 & Sub & - & + & + & ORCoNS/OSCoNS & ORCoNS & OSCoNS \\
\hline 16 & Jug & - & - & + & OSSA/OSCoNS & OSCoNS & GNB \\
\hline
\end{tabular}

Sub=subclavian; Jug=jugular; ${ }^{1}$ Oxacillin resistant Staphylococcus aureus; ${ }^{2}$ Oxacillin resistant coagulase negative staphylococci.

${ }^{3}$ Oxacillin susceptible Staphylococcus aureus; ${ }^{4}$ Oxacillin susceptible coagulase negative staphylococci; ${ }^{5}$ Gram-negative bacilli.

occlusive dressing of wounds at the insertion site of the catheter, although this practice is considered a category II procedure [10]. This fact could be the reason for the high rate of contamination found in our study.

Although there have been no previous randomized trials comparing the insertion of CVCs in the jugular and subclavian veins, some studies found a lower frequency of infection with catheterization in the subclavian than in the jugular vein $[11,12]$. However, clinicians must weigh this potential benefit against the higher risk of mechanical complications at this site. Also, observational studies made with dialysis catheters have suggested the contrary $[13,14]$.

In our study, the significant risk factors for contamination at the jugular vein were: $\geq 7$ days catheterization and $\geq 3$ invasive devices. This indicates that the patients with catheters at this site were more seriously affected and therefore required more care by the health workers.

Although central venous catheters can be contaminated by blood flow from a distal site [15], the available data show that intravascular catheters can easily become colonized externally by microorganisms from the patient's cutaneous microflora. Microorganisms can also contaminate the catheter hub where the solution feed tubing attaches to the catheter, or they may gain access to the fluid column and be infused directly into the patient's bloodstream [16]. The relative importance of colonization sources in the catheter and subsequent infection has been widely debated. Raad et al. have suggested that skin colonization is associated with shortterm catheters, while hub colonization is common in long-term catheters [17]. The strategies to make transcutaneous access to microbes more difficult, such as the use of more potent antiseptic agents, use of catheters with antimicrobials (antiseptics and antibiotics), and strict use of barrier measures during insertion, have helped to prevent the contamination of catheters and CR-BSI. Nevertheless, these practices are costly, except for the latter (maximal barrier precautions) [18]. This precaution measure was not considered in our study. 
The contamination rates in the catheters can vary according to catheter type, and some studies have demonstrated prevalence rates of bacterial contamination in CVCs of from $4.7 \%$ to $70 \%[19,20]$. In our study the contamination rate was $27 \%$ and there was a significant relationship $(\mathrm{p}=0.03)$ between contamination in the catheter tip and at the insertion site $\left(\geq 10^{2} \mathrm{CFU}\right)$.

The device that poses the greatest risk for CR-BSI today is the CVC: up to $75 \%$ of CR-BSI originate from CVCs. These catheters inserted percutaneously into the subclavian or jugular vein have shown rates of CR-BSI in the range of 3.3\%-5.1\% [21]. Our findings demonstrated a rate of CR-BSI of $2.6 \%$.

Among the microorganisms most frequently found in the CVC-related infections are: CoNS, S. aureus, Enterococcus spp., Candida spp., Corynebacterium spp, Klebsiella spp. and Enterobacter spp. [22]. Sheretz [23] demonstrated that CoNS and Staphylococcus aureus are associated with CVC infections (28\% and 16\%, respectively) and they come from the skin. In our investigation there was no quantitative difference in the numbers of the most frequent microorganisms at the jugular versus the subclavian vein. However, qualitative analysis indicated a significantly higher presence $(\mathrm{p}<0.05)$ of GNB and yeasts in the jugular vein. Some reports suggest that CVCs at this site become more infected by these microorganisms than those in the subclavian vein due to the proximity of the oropharynx, higher temperatures and difficulty in maintaining the dressing in place [09]. Staphylococci resistant to oxacillin (ORSA and ORCoNS) are increasing common, especially in tertiary and/or teaching hospitals: $34 \%$ in Brazilian institutions, 34\% in American hospitals, and about 1.8$54 \%$ in European countries [24]. Oxacillin-resistant staphylococci are commonly resistant to various antimicrobial substances, which makes it difficult to treat these types of infections; in Brazil the choice of treatment is usually empirical [25]. ORCoNS isolates can have prevalence rates of up to $85.5 \%$ [26,27], while ORSA can reach rates of $81 \%[27,28]$. The rates of $62.5 \%$ and $57.1 \%$ for ORSA and ORCoNS, respectively, were high in our series, considering that the patients were not in critical care units (Surgical). Among the risk factors for colonization/infection by multiresistant staphylococci are the use of antimicrobial substances [29,30]. However, in our study, antimicrobial substance use was not associated with an increased frequency of resistant strains at the insertion site and in the catheter tip, as about $80 \%$ of the patients that had an ORSA/ORCoNS isolate at the insertion site/catheter tip were being treated with antimicrobial substances and half of the patients with susceptible isolates were also using these substances. Though we studied relatively few patients $(n=116)$, our results suggest that there is a potential risk when not using occlusive dressings in routine care of $\mathrm{CVC}$ as more than the half of the patients had high counts of staphylococci at the insertion site of CVCs. A significant association of the type of isolate at the insertion site and in the catheter tip also confirmed the importance of the skin as a CRBSI reservoir. Although patients with catheters in the jugular vein were more severely ill and therefore had a greater bacterial contamination/infection risk when compared to those with CVC in the subclavian vein, there were no quantitative differences in the skin contamination of the insertion site nor was there a greater contamination of the catheter tips in this vein.

\section{References}

1. Egebo K., Toft P., Jakobsen C.J. Contamination of central venous catheters. The skin insertion wound is a major source of contamination. J Hosp Infect 1996;32:99-104.

2. Armstrong C.S. Clinical predictors of infection of central venous catheter used for total parenteral nutrition. Infect control Hosp Epidemiol 1990;2:71-8.

3. Maki D.G. Infections caused by intravascular devices used for infusion therapy: pathogenesis, prevention, and management. In: Infections associated with indwelling medical devices. Bisno A.L. \& Waldvogel F.A (eds). $2^{a}$ ed. American Society for Microbiology, Washington, DC. 1994, p.155-202.

4. Maki D.G., Ringer M., Alvarado C.J. Prospective randomised trial of povidine-iodine, alcohol, and chlorexidine for prevention of infection associated with central venous and arterial catheters. Lancet 1991;338:339-43.

5. Brun-Buisson C., et al. Diagnosis of central venous catheter-related sepsis. Critical level of quantitative tip cultures. Archives Intern Med, 1987;147: 873-7. 
6. National Committee for Clinical Laboratory Standards. Methods for Dilution Antimicrobial Susceptibility Tests for Bacteria That Grow Aerobically, 1997; v. 17, n.02.

7. Maki D.G, Mermel L.A.. Infections due to infusion therapy. In: Bennett J.V., Brachman P.S., eds. Hospital Infections. Boston, MA: Little Brown and Co. 1998; p.689-724.

8. Mermel L.A., Mccormick R.D., Springman S.R., et al. The pathogenesis and epidemiology of catheterrelated infection with pulmonary artery Swan-Ganz catheter: a prospective study utilizing molecular subtyping. American Journ Medicine 1991;91(Suppl 3B):S197-205.

9. Moro M.L., Vigano E.F., Lepri A.C., et al. Risk factors for central venous catheter-related infections in surgical and intensive care units. Infect Control Hosp Epidemiol 1994; $15: 253-64$.

10. Guidelines for preventing infections associated with the insertion and maintenance of central venous catheter. J Hosp Infect 2001:47(supl):S47-67.

11. Darouiche R.O., Raad I., Heard S.O. A comparison of two antimicrobial-impregnated central venous catheter. $\mathrm{N}$ Engl J Med 1999;340:1-8.

12. Goetz A.M., Wagener M.M., Miller J.M., et al. Risk of infection due to central venous catheters: effect of site of placement and catheter type. Infect Control Hosp Epidemiol 1998; 19:842-5.

13. Moss A.H., Vasilakis C., Holley I.I., et al. Use of silicone dual-lumen catheter with a dacron cuff as a long-term vascular access for hemodialysis patients. Am J Kidney Dis 1990; 16:21-5.

14. Uldall R., de Bruyne M., Besley M., et al. A new vascular catheter for hemodialysis. Am J Kidney Dis 1993;21:270-7.

15. Garland J.S., Buck R.K., Maloney P., et al. Comparison of $10 \%$ povidone-iodine and $0.5 \%$ chlorhexidine gluconate for the prevention of peripheral intravenous catheter colonization in neonates: a prospective trial. Pediatric Infectious Diseases Journal 1995;14:510-6.

16. Cooper G.L., Hopkins C.C. Rapid diagnosis of intravascular catheter-associated infection by direct gram staining of catheter segments. New Eng J Medicine 1985;312:1142-7.

17. Raad, et al. Ultrastructural analysis of indewelling vascular catheters: a quantitative relationship between luminal colonizations and duration of placement. J Infect Diseases 1993; 168: 400-7.

18. Raad I. Intravascular-catheter-related infections. Lancet 1998;351:893-8.

19. Mermel L.A. Prevention of intravascular catheter-related infections. Ann Intern Med 2000; $132: 391-402$

20. Chugh T.D., Khan Z.U. Intravascular device-related infections: antimicrobial catheters as a strategy for prevention. J Hosp Infect 2001;49:1-3.
21. Crnich C.J., Maki D.G. The promise of novel technology for the prevention of intravascular deviced-related bloodstream infection. I. Pathogenesis and short-term devices. Clin Infec Dis 2002;34:1232-42.

22. Crump J.A., Collignom P.J. Intravascular Catheterassociated infections. Eur J Clin Infect Dis 2000;19:1-8.

23. Sheretz. R.J. Surveillance for infections associated with vascular catheters. Infect Control Hosp Epidemiol 1996; $17: 746-52$.

24. Diekema D.J., Pfaller M.A., Schimitz F.J. et al. Survey of infections due to Staphylococcus species: frequency of occurrence and antimicrobial susceptibility of isolates collected in the United States, Canada, Latin America, Europe, and the Western Pacific Region for the SENTRY antimicrobial surveillance program, 1997-1999. Clin Infect Dis 2001;32(supl):S114-32.

25. Couto H.G..., et al. Vancomycin use in a Brazilian University Hospital: Comparison with Hospital Infection Control Practices Advisory Committee Guidelines. Brazilian Journal Infectious Diseases 1999;3:139-43.

26. Frebourg N.B., Cauliez B., Lemeland J.F. Evidence for nasal carriage of methicillin-resistant Staphylococci colonizing intravascular devices. J Clin Microbiol 1999;37:1182-5.

27. Gray J.W., George R.H. Is the incidence of MRSA bacteraemia representative of the rate of MRSA infection in general? J Hosp Infect. 2001;49(1):79.

28. Stamm AM, Long MN, Belcher B. Higher overall nosocomial infection rate because of increased attack rate of methicillin-resistant Staphylococcus aureus. Am J Infect Control 1993;21:70-4.

29. Sadoyama G., Gontijo Filho P.P. Risk Factors for methicillin resistant and sensitive Staphylococcus aureus infection in a Brazilian university hospital. Brazilian Journal Infectious Diseases 2000;4:135-43.

30. Boyce J.M. Coagulase-negative staphylococci. In: C. Glen Mayhall, M.D. (Ed) Hospital Epidemiology and Infection Control. Willians \& Wilkins Company, Baltimore, USA. 1996. p. 306-34. 